\title{
Broadband spectral investigations of SGR J1550-5418 bursts
}

\author{
Lin Lin and Ersin Göğüş \\ Sabancı University, Faculty of Engineering and Natural Sciences, Orhanlı- Tuzla, \\ İstanbul 34956, Turkey \\ email: linlin@sabanciuniv.edu
}

\begin{abstract}
We present the results of our broadband $(0.5-200 \mathrm{keV})$ spectral analysis of 42 SGR J1550-5418 bursts simultaneously detected with the Swift/X-ray Telescope (XRT) and the Fermi/Gamma-ray Burst Monitor (GBM), during the 2009 January active episode of the source. We find that, on average, the burst spectra are better described with two blackbody functions than with the Comptonized model. Thus, our joint XRT/GBM analysis clearly shows for the first time that the SGR J1550-5418 burst spectra might naturally be expected to exhibit a more truly thermalized character, such as a two-blackbody or even a multi-blackbody signal. We also studied the spin phase of the XRT burst emission, which indicate that the burst emitting sites on the neutron star need not to be co-located with hot spots emitting the bulk of the persistent X-ray emission and the surface magnetic field of SGR J1550-5418 is likely non-uniform over the emission zone.
\end{abstract}

Keywords. stars: neutron, pulsars: individual (SGR J1550-5418, 1E 1547.0-5408, PSR J15505418), X-rays: bursts

\section{Introduction}

Magnetars are isolated neutron stars possessing extreme magnetic fields over $10^{14} \mathrm{G}$, observed as Soft Gamma Repeaters (SGRs) and Anomalous X-ray Pulsars (AXPs). Besides being bright X-ray sources, SGRs and AXPs emit intense bursts in hard X-rays / soft $\gamma$-rays on a highly unpredictable frequency. A typical burst from magnetars lasts for $\sim 100 \mathrm{~ms}$ with the peak luminosity of $10^{38} \sim 10^{41} \mathrm{erg} \mathrm{s}^{-1}$. Its spectrum is equally well described with a Comptonized model (COMPT) or the sum of two blackbody functions $(\mathrm{BB}+\mathrm{BB})$ in $8-200 \mathrm{keV}$ (Lin et al. 2011, van der Horst et al. 2012). These two models, which have very different physical origin (thermal or non-thermal) cannot be distinguished in the GBM energy range. However, they have large dispersion in the lower energy band below $10 \mathrm{keV}$, making the model discrimination possible.

\section{Sample selection}

The unique spectral and temporal capabilities of the XRT Windowed Timing mode have allowed us to extend the GBM spectral coverage down to the X-ray domain $(0.5-10$ $\mathrm{keV}$ ). We found 42 SGR J1550-5418 bursts simultaneously detected with the Swift/XRT and the Fermi/GBM during its 2009 January active episode. Figure 1 exhibits the lightcurve and the spectrum of one of the simultaneous events.

\section{Results}

We fit all 42 simultaneously bursts with both COMPT and BB+BB models using only GBM data and joint XRT-GBM data. On average, BB+BB model fits better than 

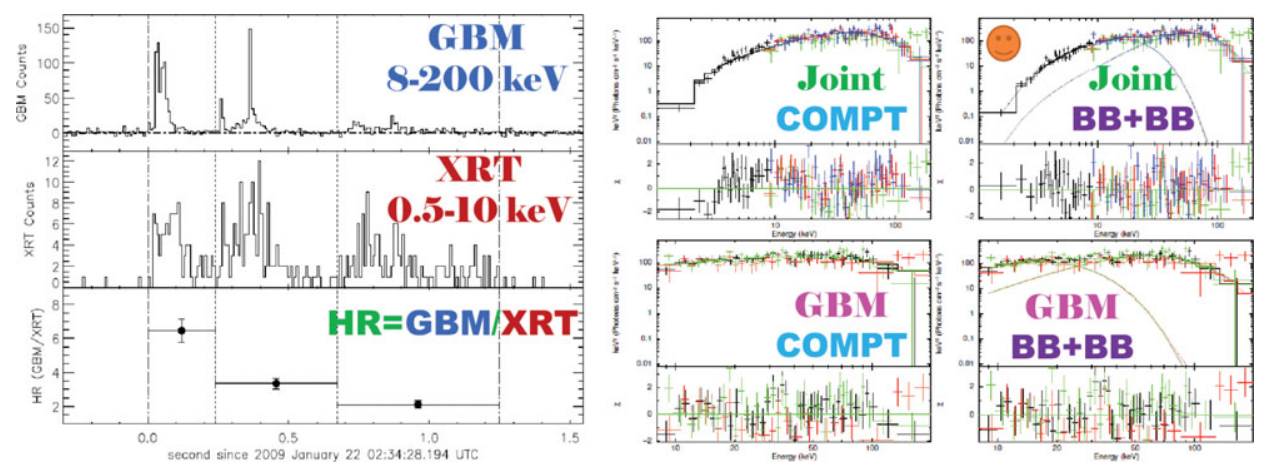

Figure 1. Left: Lightcurves of a burst simultaneously observed with GBM and XRT with a temporal resolution of $8 \mathrm{~ms}$. The bottom panel shows the hardness ratios of the three subintervals indicated with the dotted lines. Right: The XRT-GBM joint fit and GBM data only fit spectra of the same burst.
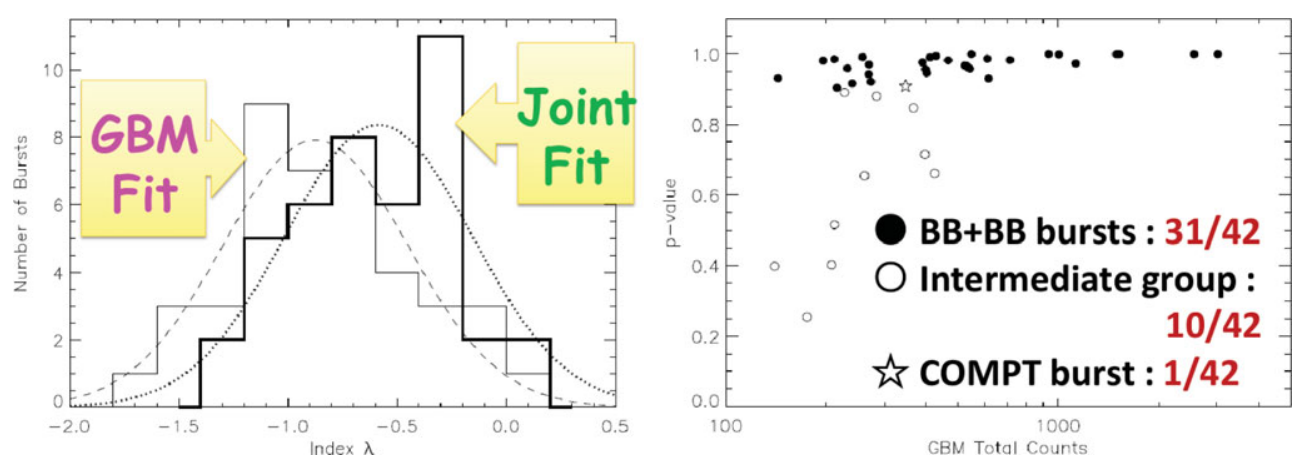

Figure 2. Left: The distribution of the COMPT power law index of the GBM data only fit and the joint fit. Right: Plot of the p-value versus the total counts as seen with GBM.

COMPT. First of all, BB + BB model fits provide less systematic residuals (see joint fit spectra in Figure 1) and smaller average reduced $\chi^{2}$ values. Secondly, the mean value of the COMPT index distribution from joint analysis is $-0.58 \pm 0.09$, much harder than GBM data only result $(-0.87 \pm 0.05)$, as shown in the left panel of Figure 2 . This indicates that GBM data only fits with COMPT model may over estimate the low energy emission. Finally, since the COMPT model has one less parameter than the BB+BB function and they are not nested, we performed extensive simulations for each of the 42 bursts to determine the significance of the model preference (see Lin et al. 2012 for more details). We selected the model with smaller $\chi^{2}$ as seed model and simulate 10000 spectra with the seed model. Then we fit all simulated spectra with both COMPT and BB+BB models. We then calculated the probability $(\mathrm{P})$ of the simulated spectra have a smaller $\chi^{2}$ fit with the seed model. We defined the seed model significantly better than the other one in case of $\mathrm{P}>90 \%$. For 31 bursts out of 42 the BB+BB model fits significantly better than the COMPT. The bright bursts in our sample prefer the $\mathrm{BB}+\mathrm{BB}$ model. The right panel of Figure 2 presents the relation between $\mathrm{P}$ and the total counts of bursts in $8-200 \mathrm{keV}$ band.

We further studied the properties of $31 \mathrm{BB}+\mathrm{BB}$ bursts. The temperature of two blackbody components are $4.4 \pm 0.2 \mathrm{keV}$ and $16.0 \pm 0.4 \mathrm{keV}$, consistent with those from other magnetar bursts. The energy emitted from hot blackbody is twice the energy from the cool one. Assuming the distance to SGR J1550 - 5418 as $5 \mathrm{kpc}$, we calculate the emission 

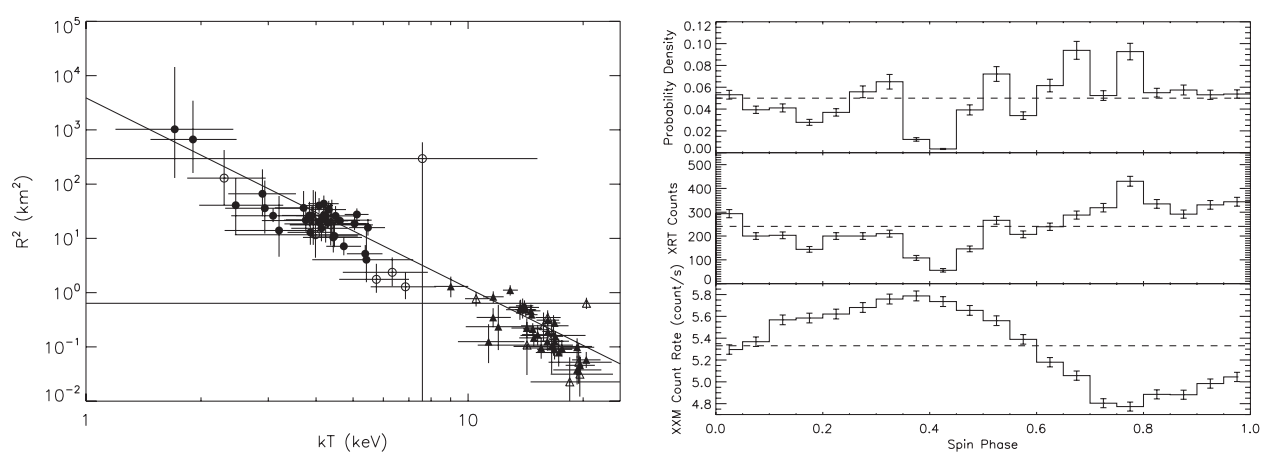

Figure 3. Left: The correlation between the emission area and the temperature of both blackbody components. The BB $+\mathrm{BB}$ bursts are marked with solid symbols, while the empty ones are present the intermediate bursts. The hot and cool blackbody component are exhibit with triangles and dots respectively. The solid line presents the best power law fit with both blackbody components, $R^{2} \propto(k T)^{-3.5}$. Right: The phase distribution of XRT counts from $31 \mathrm{BB}+\mathrm{BB}$ bursts.

area for each blackbody component. The correlation between the emission area and the temperature through cool and hot component is similar to that of a single blackbody with a certain flux (Figure 3 left panel).

To better understand the $\mathrm{BB}+\mathrm{BB}$ behavior and uncover its relation with the spin properties of SGR J1550-5418, we investigated the phase characteristics of the $31 \mathrm{BB}+\mathrm{BB}$ bursts (see the right panel of Figure 3). We selected all XRT counts collected during 31 burst intervals and calculated the spin phase for each burst count using the appropriate spin ephemeris of epoch (MJD) 54854 as reported by Dib et al. (2012). To ensure that the distribution is not dominated by the excessive counts of the brightest bursts, we also calculated the probability density for each phase bin, which is the average of the normalized (by total counts) phase distributions for all bursts. We find that the probability distribution of the burst counts is not uniform over the spin phase of SGR J1550-5418 and the deviation from the mean probability is significant (RMS $=0.021 \pm 0.001)$. Compared with the persistent emission phase profile obtained using contemporaneous XMM observations, the phase probability density function is marginally anti-correlated with the persistent emission phase profile in our burst sample. This indicates that the burst emission regions on the neutron star surface are not necessarily associated with the site persistently emitting in X-rays (typically a BB with a temperature of $0.5 \mathrm{keV}$ ). This is in agreement with the crustal fracturing mechanism for SGR bursts (Thompson \& Duncan 1995) as any portion of the solid crust can fracture if the magnetic stress built up is near the threshold to rupture. We also find that the burst probability of some spin phases in SGR J1550-5418 is higher. This could be attributed to a non-uniform surface magnetic field, with some regions having larger magnetic stresses than others.

\section{Acknowledgements}

LL acknowledges support from the Turkish Academy of Sciences.

\section{References}

Dib, R., Kaspi, V. M., Scholz, P., \& Gavriil, F. P. 2012, ApJ, 748, 3

Lin, L., Kouveliotou, C., Baring, M. G., et al. 2011, ApJ, 739, 87

Lin, L., Göğüs, E., et al. 2012, ApJ, 756, 54

Thompson, C. \& Duncan, R. C. 1995, MNRAS, 275, 255

van der Horst, A. J., Kouveliotou, C., Gorgone, N. M., et al. 2012, ApJ, 749, 122 